DOI: $10.35643 /$ Info.26.2.9

Artículo original

\title{
Universidad, estudiantes y comunidad virtual de aprendizaje: WhatsApp como promotor para la construcción de un nuevo espacio tecnológico, geográfico y social
}

\section{University, students, and virtual learning community: WhatsApp as a driver of a new technological, geographical, and social space \\ Universidade, alunos e comunidade virtual de aprendizagem: o WhatsApp como promotor da construção de um novo espaço tecnológico, geográfico e social}

\author{
Mauricio Olivera ${ }^{1}$ ORCID 0000-0003-0018-1940 \\ Varenka Parentelli ${ }^{1}$ ORCID 0000-0003-2033-7949
}

${ }^{1}$ Facultad de Información y Comunicación, Uruguay. San Salvador 1944,
Montevideo, Uruguay. Correos electrónicos: mauricio.olivera@,fic.edu.uy y
varenka.parentelli@fic.edu.uy.

\section{Resumen}

Este estudio indaga sobre las apropiaciones que los estudiantes hacen de las tecnologías de la información y la comunicación para acceder a los materiales de estudio en internet, teniendo en cuenta sus prácticas digitales en las redes sociales, a partir de una encuesta autoadministrada $(n=1300)$, grupos de discusión $(n=22)$ y una entrevista en profundidad a la coordinadora del Entorno Virtual de Aprendizaje de la Facultad. La combinación y triangulación de estas técnicas confirman que los estudiantes han generado un nuevo escenario tecnológico y reticular a partir del uso de WhatsApp en la mediación comunicacional e informacional, transformando sus prácticas y estrategias de estudio, el acceso y la apropiación de los materiales de estudio (formales e informales) y de la información institucional. Los hallazgos, las líneas futuras de investigación y las implicaciones se discuten en el contexto de una comunidad virtual de aprendizaje 
que denominamos: nuevo espacio tecnológico, geográfico y social.

Palabras clave: WHATSAPP; UNIVERSIDAD; COMUNIDADES VIRTUALES DE APRENDIZAJE Y EDUCACIÓN SUPERIOR; ESPACIO TECNOLÓGICO, GEOGRÁFICO Y SOCIAL.

\begin{abstract}
This study researches the IT appropriations that students make to access online study materials, considering their digital practices on social networks from a selfadministered questionnaire $(\mathrm{N}=1300)$, focus groups $(\mathrm{N}=22)$, and an in-depth interview with the school's Virtual Learning Environment coordinator. The combined application of these techniques confirms that students have created a new technological and netlike scenario by using WhatsApp to communicate and mediate information, transforming their study practices, appropriation of institutional information and formal and informal study materials. Findings, future research and implications are discussed in the context of a virtual learning community that we call: new technological, geographical and social space.

Keywords: WHATSAPP; UNIVERSITY; VIRTUAL LEARNING COMMUNITIES AND HIGHER EDUCATION; TECHNOLOGICAL, GEOGRAPHICAL AND SOCIAL SPACE.
\end{abstract}

\title{
Resumo
}

Esta pesquisa investiga as apropriações que os alunos fazem das TIC para o acesso a materiais de estudo na internet, tendo em conta as suas práticas digitais nas redes sociais, a partir de inquérito autoaplicável $(\mathrm{N}=1300)$, grupos de discussão $(\mathrm{N}=22)$ e-entrevista em profundidade com o coordenador do Ambiente Virtual de Aprendizagem da Faculdade. A combinação e triangulação dessas técnicas confirmam que os alunos geraram um novo cenário tecnológico e reticular a partir do uso do WhatsApp, na mediação comunicacional e informacional, transformando suas práticas e estratégias de estudo, acesso e apropriação de materiais de estudo. Estudo (formal e informal) e informações institucionais. Achados, futuras linhas de pesquisa e implicações são discutidos no contexto de uma comunidade virtual de aprendizagem que chamamos de: novo espaço tecnológico, geográfico e social.

Palavras-chaves: WHATSAPP; UNIVERSIDAD; COMUNIDADES VIRTUAIS DE APRENDIZAGEM E ENSINO SUPERIOR; ESPAÇO TECNOLÓGICO, GEOGRÁFICO E SOCIAL.

Fecha de recibido: 15/07/2021

Fecha de aceptado: 26/08/2021 


\section{Introducción}

Los estudiantes universitarios pasan gran parte de su tiempo en internet [1] y son quienes más utilizan las redes sociales (Azizi, Soroush y Khatony, 2019). Según el estudio El Perfil del Internauta Uruguayo (Radar, 2019), el $98 \%$ de los internautas que tiene un dispositivo móvil instaló WhatsApp en su pantalla inicial y el $93 \%$ aseguró que lo cliqueó para usarlo en la última semana. En Uruguay, nueve de cada diez usuarios de esta plataforma la utilizan para comunicarse con los amigos, familiares, colegas, etc., y cuatro de cada diez usuarios integran cinco o más grupos (en promedio). La edición 2020 del mismo estudio (Radar, 2020) señala que el $93 \%$ de los uruguayos — de todas las edades - son usuarios de internet con un tiempo promedio de 5,6 horas y que el dispositivo más usado es el smartphone (un $82 \%$ de los internautas lo utilizan). Asimismo, se constata que hay una tendencia al crecimiento de la conexión a internet de los jóvenes, así como el en uso de las tecnologías de redes sociales (en inglés, social networking technologies o SNT [2]) para compartir y buscar información (Olivera, 2020a). Se optó, en este artículo, por utilizar la sigla SNT, tal como se la conoce en la literatura anglosajona, debido a que es así como se lo identifica con la teoría de las redes sociales. Esta observa las relaciones sociales en términos de nodos y vínculos. Los nodos son los actores individuales dentro de las redes y los vínculos son las relaciones entre los actores. Puede haber muchos tipos de vínculos entre los nodos. En su forma más simple, una red social es un mapa de todos los vínculos relevantes entre los nodos que se estudian. La red también se puede utilizar para determinar el capital social de actores individuales.

Algunos estudios señalan que los sitios de redes sociales juegan un papel fundamental en la educación, debido a que los estudiantes tienen múltiples oportunidades para mejorar el aprendizaje y acceder a la información más reciente al conectarse con grupos de aprendizaje y otros sistemas educativos (Martín Barbero, 2006; Greenhow y Robelia, 2009a; Scolari, 2018). Los estudiantes también intercambian información al conectarse entre ellos (Olivera, 2020a), lo que puede tener, según algunos autores, un impacto positivo en los resultados del aprendizaje de los estudiantes (Yu, Tian, Vogel y Kwok, 2010). No obstante, las redes sociales pueden tener un impacto negativo en la salud mental (Kolhar, Raisa 
y Abdalla, 2021).

El uso de las SNT con fines de aprendizaje se sigue debatiendo en el ámbito académico. Para algunos, se relaciona negativamente con el rendimiento académico (Hew, 2011; Junco, 2012; Junco y Cotten, 2012) y, para otros, lograron una transformación y un cambio en la enseñanza tradicional a partir del aprendizaje colaborativo (Bingham y Conner, 2015; Collins y Halverson, 2009; Greenhow y Robelia, 2009b; Wegerif, 2012). El aprendizaje colaborativo se define como una estrategia basada en un proceso donde prima la interacción entre pares -en este caso, los estudiantes-, quienes diseñan y organizan las estructuras de interacciones y controlan los aspectos que inciden en sus procesos de aprendizaje (Collazo y Mendoza, 2006). No obstante, para que el aprendizaje sea efectivamente colaborativo, los pares deben pertenecer a un mismo nivel, tener un objetivo común y trabajar en conjunto (Dillenbourg y Baker, 1996). Puede considerarse que el uso de las SNT, desde una perspectiva colaborativa, incida en el desarrollo de estrategias para el aprendizaje, por lo que es importante tener en cuenta que no es un mecanismo simple (Dillenbourg, 1999). Es decir, si bien existe una mayor probabilidad de interacción que podría generar aprendizaje, esto no siempre es así. La colaboración no es condición suficiente para lograr un aprendizaje, ya que en ese proceso existen, además, dispositivos tales como la lectura, la construcción, la predicción que estimula la inducción, la deducción y la compilación; así como otros dispositivos adicionales (explicaciones, desacuerdos, regulación), a través de los cuales emergen mecanismos cognitivos como la internalización, la extracción y el conocimiento (Zañartu, 2003). Las TIC incorporan nuevas herramientas sociocognitivas de creación y apropiación sociocultural a través de las cuales se generan entornos de interacción que podrían conducir a la construcción de conocimiento y de aprendizaje (Cardozo, 2010). Los entornos generados por las tecnologías reúnen características que potencian la colaboración, tales como la interactividad, la ubicuidad y el sincronismo. Aunque la mediación tecnológica no es una conditio sine qua non para que se geste el aprendizaje colaborativo, es importante registrar que el proceso de interacción entre las personas y las TIC es el responsable, en gran parte, del nacimiento de la sociedad del conocimiento. Por eso, es necesario tener en cuenta el fenómeno 
conocido como Computer Supported Collaborative Learning (CSCL) (Zañartu, 2003, p. 4). Según esta lógica, se crean las comunidades virtuales (CV), constituidas en torno a un interés, con el fin de generar una activa participación y compartir información entre sus miembros (Gairín, 2006). Las personas se agrupan en una comunidad porque desean adquirir e intercambiar conocimientos sobre un tema de interés (necesidad de autorrealización), pero también para relacionarse con aquellos que comparten sus mismos intereses (necesidad de pertenencia) (Silvio, 1999).

No obstante, también hay investigaciones que advierten sobre los trastornos por el uso de los smartphones o trastorno de la comunicación en internet, término utilizado por Matthias Brand, Kimberly Young, Christian Laier, Klaus Wölfling y Mark Potenza (2016) y por Montag, et al. (2018). El trabajo de Peng Sha, Rayna Sariyska, René Riedl, Bernb Lachmann y Christian Montag (2019) señala lo siguiente: «La superposición entre el concepto de trastorno por uso de WhatsApp y el trastorno por uso de teléfonos inteligentes es sólida», y agrega que «WhatsApp juega un papel importante para comprender el trastorno por uso de teléfonos inteligentes también en los hombres, pero factores adicionales como el trastorno del juego también pueden ser relevantes» (p. 6).

Otros autores, en cambio, son más cautos y entienden que aún hay que profundizar en las dimensiones y complejidades del fenómeno de las SNT, tensionando así la simplificación de la discusión en relación con el impacto o los estudios que solo atienden a los éxitos o aspectos positivos de las SNT (Piotrowski, 2015; Selwyn y Stirling, 2016; Livingstone y Sefton-Green, 2016; Bouton, Bar Tal y Asterhan, 2021).

El objetivo de este artículo es contribuir a esta discusión a partir de un estudio que documenta las apropiaciones que realizan los estudiantes universitarios de las SNT para acceder a los materiales de estudio en internet, en relación con sus prácticas digitales y con sus redes sociales, dentro de la Facultad de Información y Comunicación de la Universidad de la República en Uruguay. 


\subsection{Usos académicos de internet en educación superior}

En los últimos años se publicaron varias investigaciones sobre las TIC en relación con la educación (Selwyn y Stirling, 2016; Cobo, 2016; Pardo y Cobo, 2020). En general, se centran en cómo los estudiantes aprovechan las SNT dentro de la vida universitaria (Amador y Amador, 2014; DeAndrea, Ellison, LaRose, Steinfield y Fiore, 2012; Wodzicki, Schwämmlein y Moskaliuk, 2012) y abordan las innovaciones y formación de profesorado para articular las SNT con el currículo oficial del curso o lo que Moore (1989) llamó interacciones entre profesorestudiante (Dabbagh y Kitsantas, 2012; Laru, Näykki y Järvelä, 2012; Molinillo, Anaya-Sánchez, Aguilar-Illescas y Vallespín-Arán, 2018; Rodés, Gewerc-Barujel y Llamas-Nistal, 2019).

Sin embargo, el uso de las SNT en relación con el estudio académico ha sido poco explorado científicamente. Algunos trabajos recientes (Smith, 2016; Olivera, Morales, Passarini y Correa, 2017; Olivera, 2020a; Olivera, 2021) indican que la mayoría de las interacciones de los estudiantes en las SNT con fines de estudio son estudiante-estudiante, con poca o fallida participación docente-estudiante. Curiosamente, aún se sabe poco acerca de las interacciones de los estudiantes en las SNT con fines de estudio o de cómo y a qué materiales acceden, cómo valoran los materiales que obtienen en internet y qué tipos de materiales producen y comparten.

En Uruguay, la Universidad de la República (Udelar) comenzó un proceso de integración de TIC en la enseñanza y en el aprendizaje a partir del año 2000 y, en el 2008, se incorporó el Entorno Virtual de Aprendizaje (EVA). En el año 2019, se identificaron 283803 usuarios en el EVA: a) el $93 \%$ de los estudiantes de grado ya tenían usuario en esa plataforma; b) el $55 \%$ se consideraba un usuario frecuente o muy frecuente; c) para el $56 \%$ fue la fuente principal de recursos educativos para estudiar, y d) el $65 \%$ manifestó que el entorno virtual facilitó un mejor rendimiento académico (Universidad de la República, 2019a, 2019b). El Programa de Entornos Virtuales de Aprendizaje de la Udelar (ProEvA), responsable de la implementación, gestión y desarrollo del EVA, entendió en 2019 que el proceso iniciado en el año 2008 habría alcanzado una universalización de 
los EVA.

Otro estudio más reciente del Proeva (Universidad de la República, 2020), en el contexto de pandemia provocada por la llegada del virus SARS-CoV-2, identificó un aumento promedio del $18 \%$ de usuarios en EVA y del $28 \%$ de creación de cursos. Sin embargo, pese al incremento del uso del EVA debido a la suspensión de las clases presenciales, los recursos más utilizados de la plataforma fueron los mensajes para la comunicación docente-estudiante y los cuestionarios para la evaluación de los aprendizajes. Este estudio señala además que, en el primer semestre del 2020, las visitas al EVA central, donde se alojan varias carreras de la Udelar, aumentaron un 130 \% (5 632082 visitas), en comparación con el primer semestre de 2019. Sin embargo, estos estudios no avanzan en aquellos aspectos relacionados con las SNT y el rendimiento académico, la relación entre estudiantes y docentes o la influencia e incidencia que estas tienen en sus procesos de aprendizaje.

En esta investigación se propuso como objetivo avanzar en el conocimiento y el alcance, así como en las características del uso de SNT, en relación con el acceso de materiales de estudio en internet dentro del entorno educativo superior, a partir de una muestra censal autodirigida de los estudiantes activos de la FIC, Udelar, y con un enfoque cualitativo.

\subsection{Configuración de un nuevo espacio tecnológico, geográfico y social}

En un estudio cualitativo exploratorio reciente, desarrollado en la sede del Centro Universitario Regional (Cenur) Litoral Norte de la Udelar, se documentó cómo la intervención de nuevas formas de comunicación (SNT), sobre todo a través de WhatsApp, produjo cambios que alteraron y tensionaron las formas tradicionales de relacionamiento de los estudiantes universitarios, generando nuevas necesidades tecnosociales entre los jóvenes (Olivera, 2020a). Las prácticas tecnosociales participativas que constituyeron e instituyeron estos estudiantes son gestionadas a partir de sistemas propios de gobernanza, dentro de lo que definimos como un nuevo espacio tecnológico, geográfico y social (TGS), que 
condensa y establece lógicas y temporalidades diversas, concibiendo, a la vez, fenómenos de integración y de exclusión informacional y comunicacional (Olivera, 2019). En ese contexto, se observaron las formas particulares de las prácticas comunicativas, sociales y tecnológicas de los actores universitarios que ahora interactúan entremezclando lógicas y temporalidades diversas, condensando, al mismo tiempo, una nueva relación con el espacio, el tiempo y el lugar. En ese escenario, se detectó un actor clave: el delegado/referente estudiantil, quien actúa como un nodo en la red comunicacional universitaria por el que circula un flujo tecnoinformacional que abarca a los estudiantes, pero también a los docentes y a la institución. Tal como se desprende del estudio, las nuevas prácticas resignifican los espacios tradicionales y digitales de producción y circulación de la información.

La referencia a los espacios TGS permite entender las profundas transformaciones en las formas de concebir la relación espacio-tiempo y los cambios en curso de la experiencia humana, puesto que los términos geográfico y social son conceptos que las personas tienen del mundo, que se emplean en la vida cotidiana y en las prácticas científicas para orientar, demarcar, diferenciar y reducir la complejidad para dar sentido a varios fenómenos. Hay una conexión dialéctica entre ambos, ya que no existe lo social sin una dimensión geográfica ni tampoco una construcción geográfica sin una dimensión social.

La revolución digital permitió que la tecnología atraviese lo geográfico y lo social, permitiendo novedosas lógicas y formas de producción, circulación y apropiación de la información en un contexto de cambio tecnológico continuo y dinámico. En la actualidad, las TIC permiten crear entre los actores sociales nuevas prácticas y lógicas de participación configuradas por relaciones nuevas y múltiples entre el espacio, el tiempo y el lugar (Olivera, 2013). Los espacios TGS están mediados por prácticas socioculturales y por características tales como la distancia, el territorio, la pertenencia, la identidad y la sociabilidad. Son nodos, espacios donde confluyen parte de las conexiones de otros espacios reales o abstractos que comparten sus mismas características y que, a su vez, también son nodos que conforman una red. Asimismo, configuran sistemas heterárquicos [3] y creativos. McCulloch (1945), quien describió la conexión heterárquica por 
primera vez, entendió que, en un sistema cualquiera, existen relaciones tanto jerárquicas como heterárquicas y que, en estas últimas, hay una situación de interdependencia entre niveles o subsistemas diferentes, en los cuales se despliegan procesos diversos y de manera concurrente. La dinámica de interacciones dentro del sistema heterárquico implica que no hay un único sistema gobernante, sino que cada subsistema ejerce cierta influencia sobre los demás. La ausencia de un subsistema gobernante en las heterarquías se debe a que en ellas existen múltiples accesos, múltiples vínculos y múltiples determinaciones entre los distintos subsistemas.

Por lo tanto, estos espacios TGS son un lugar de observación clave de las nuevas tecnosociabilidades, de las relaciones que surgen y se difunden entre los actores sociales, así como de sus prácticas tecnológicas, a través de las cuales se expresan sentidos y significados (Olivera, 2020a). En ese contexto, internet cumple una función primordial: es la herramienta que permite esas relaciones y habilita los espacios TGS, donde circula la compleja relación entre distancias, territorios y sentidos que orientan las prácticas de los actores sociales (Olivera, 2013). El desafío es observar y analizar la constitución de tales prácticas a partir de la relación tecnológica, geográfica y social que habilita internet.

Sin embargo, estos hallazgos no dieron cuenta de cómo las nuevas formas SNT, a partir de las apropiaciones de las TIC, incidían en las formas de acceso y circulación de los materiales de estudio en el ámbito estudiantil universitario. Es decir, cuáles eran las prácticas relacionales tecnocomunicacionales de los estudiantes universitarios para la apropiación de los materiales de estudio en el marco de ese nuevo espacio TGS (y de qué tipo). Por lo anterior, este trabajo se propuso indagar en ese espacio de vacancia en el marco de la FIC.

\section{Metodología}

El presente trabajo forma parte de una línea de investigación sobre el uso, apropiación y prácticas digitales de los estudiantes con fines de estudio, en la que se combinan diversas técnicas metodológicas (encuestas, análisis de contenido, grupos focales y entrevistas en profundidad), principalmente dirigidas a los estudiantes de educación superior. En este artículo se pone el foco, 
específicamente, en la apropiación de los materiales de estudio a partir de las SNT en el entorno de educación superior en la Facultad de Información y Comunicación de la Universidad de la República, Uruguay. En este marco, se buscó explorar el fenómeno de apropiación e intercambio de materiales de estudio entre los estudiantes universitarios de toda una facultad, a partir de una metodología mixta y secuencial que trianguló los datos obtenidos.

\subsection{Participantes y materiales}

El diseño metodológico de este trabajo, tomando como unidad de análisis a los estudiantes de la FIC, Udelar, durante el período 2018-2019, consistió en dar cuenta de dos grandes dimensiones: los espacios relacionales sociocomunicacionales y los espacios socioeducativos. Se optó por excluir de la muestra a los estudiantes iniciales, de primer año, porque se consideró que no tenían incorporadas las prácticas y los usos tecnológicos en relación con el tiempo y con la apropiación de los materiales de estudio en la FIC. El criterio de selección fue que los estudiantes hubieran atravesado tres períodos de exámenes (julio y diciembre de 2018 y febrero de 2019), debido a que en esas instancias se produce el mayor intercambio de información y relacionamiento de los estudiantes, tal como se desprende de la investigación «Plataformas virtuales: ¿Herramientas para el aprendizaje? Las diferencias entre ADÁN y EVA» (Olivera et al., 2017).

Las dimensiones del formulario utilizado para realizar la encuesta se dividieron en tres secciones: 1) Acceso y uso general de TIC; 2) Canales de comunicación en la FIC, y 3) Habilidades. A los efectos de segmentar a los estudiantes censados, se tomó en cuenta el relevamiento del perfil sociodemográfico básico. El N arrojó, aproximadamente, entre 1200 y 1300 estudiantes activos, comprendidos entre segundo y cuarto año de su carrera. A los efectos de probar el instrumento, se realizó una prueba del test en marzo a 150 estudiantes y se alcanzó a encuestar un $\mathrm{N}$ real total de 765 estudiantes durante abril y mayo de 2019.

Se observó, en particular, la regularidad y el tipo de uso de las herramientas TIC utilizadas por los estudiantes de dos generaciones de la FIC durante los tres períodos de exámenes, con el fin de analizar si existían comportamientos similares entre ambas generaciones. Para ello, se aplicaron técnicas cuantitativas, como la 
encuesta y el análisis de datos secundarios, así como también técnicas cualitativas, como la entrevista en profundidad y los grupos de discusión.

Como primera acción, se elaboró una encuesta en línea autoadministrada dirigida a los estudiantes con el objetivo de explorar los espacios de intercambio comunicacionales virtuales dispuestos por ellos mismos a partir de variables tales como preferencias y uso de las plataformas, frecuencia de utilización, búsqueda de información, interés en la información difundida por la universidad, motivaciones y tiempo destinado al uso. Dicha encuesta se implementó mediante sucesivas barridos[4] en las asignaturas obligatorias y electivas de las tres licenciaturas de la FIC: Comunicación, Bibliotecología y Archivología. Se tomó como universo de estudio a los estudiantes activos que, según la definición institucional, son todos aquellos que hayan realizado al menos una de las siguientes actividades dentro de un período estimado de dos años: a) inscribirse a un curso curricular; b) haber exonerado una asignatura, o c) tener un examen rendido.

Con el fin de triangular con el análisis cuantitativo para profundizar en los aspectos subjetivos, tales como significados y apropiaciones de las plataformas virtuales de estudio y percepción de las carreras de la FIC, se aplicaron dos técnicas cualitativas: la entrevista en profundidad y los grupos de discusión. El criterio de selección que se consideró como factor relevante fue el avance en la carrera, a los efectos de indagar en la experiencia transitada en las acciones desarrolladas para el estudio y, en ello, el sentido del uso de las TIC. Para lo anterior, se diseñó una pauta de entrevista y una pauta para los grupos de discusión, que tomaron como antecedentes algunos de los resultados de la encuesta y aspectos emergentes de interés. Estas pautas se elaboraron de acuerdo a los perfiles de apropiación digital de los estudiantes, lo que permitió profundizar y complementar la información más relevante, así como construir los datos que dieron cuenta del objetivo general del proyecto (Olivera, 2020b).

Se convocó a estudiantes de los semestres más avanzados: Ciclo de Profundización (5..$^{\circ}$ semestre) y Ciclo de Graduación $\left(6 .^{\circ}, 7 .^{\circ}\right.$ y $8 .^{\circ}$ semestre). Para la convocatoria, a partir de la estrategia bola de nieve [5], se los contactó a través del Espacio Virtual de Aprendizaje (EVA), así como por otros canales informales, como los grupos de estudiantes en WhatsApp. Se realizaron cuatro grupos de 
discusión y dos entrevistas en profundidad con estudiantes de distintos ciclos y carreras. Asimismo, se concretó una entrevista en profundidad con la articuladora de la plataforma EVA de la FIC que ocupó el cargo durante el período estudiado.

\section{Resultados y discusión}

La gran mayoría $(72,2 \%)$ de los encuestados, a quienes se les pidió que marcaran hasta tres opciones sobre el uso habitual que realizaban de internet, señalaron «para estudiar» (véase figura 1); porcentaje solo superado por navegar y SNT $(87,1 \%)$. Se tomó la variable de la edad y se recodificó en tabulación cruzada con el uso habitual (fig. 2). Los datos muestran que el uso de las SNT para estudiar no varía de forma significativa según la edad (18 a 20, 21 a 24, 25 a 30, más de 31 años). La diferencia entre el uso de internet según la edad sí se observa en la búsqueda de noticias o tareas laborales (véase figura 2). Otro dato relevante es el dispositivo que utilizan los estudiantes habitualmente para conectarse a internet: smartphone $(49,3 \%)$ y computadora $(43,1 \%)$. La variable frecuencia de conexión de los estudiantes por edad (véase figura 3) tampoco arroja datos significativos.

En síntesis, los datos cuantitativos indican que: a) los estudiantes universitarios tienen una alta conectividad desde sus smartphones (todos los encuestados tienen un dispositivo móvil); b) utilizan internet para acceder a sus materiales de estudio, y c) la edad de los estudiantes no es una variable significativa.

Figura 1: Porcentajes de estudiantes según el uso de internet informado
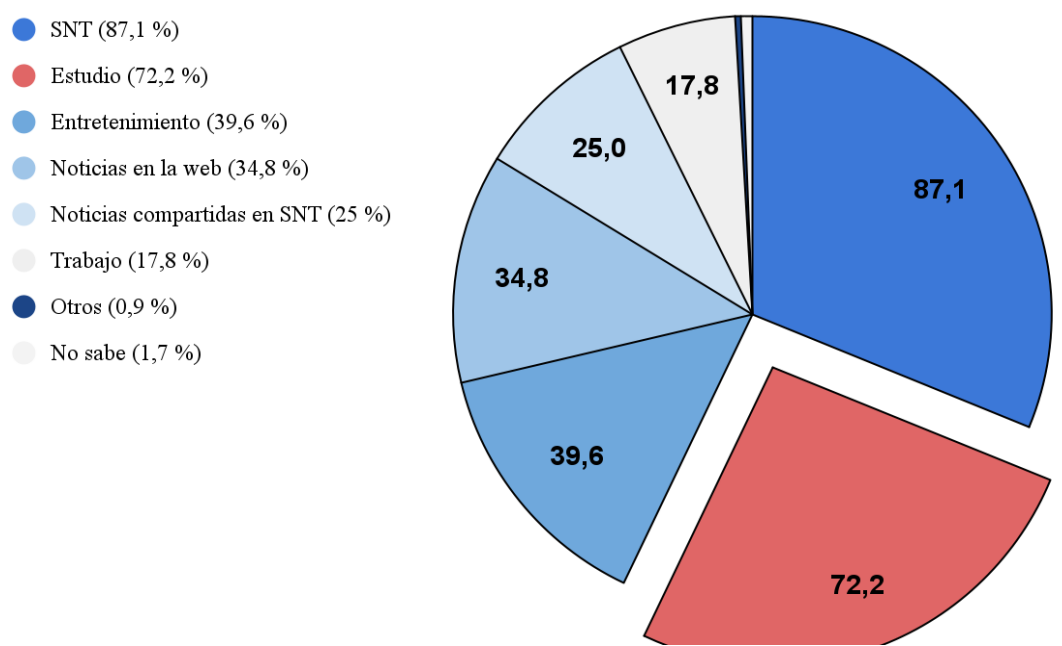

Fuente: elaboración propia. 
Figura 2: Tipos de uso de internet de los estudiantes según la edad 40

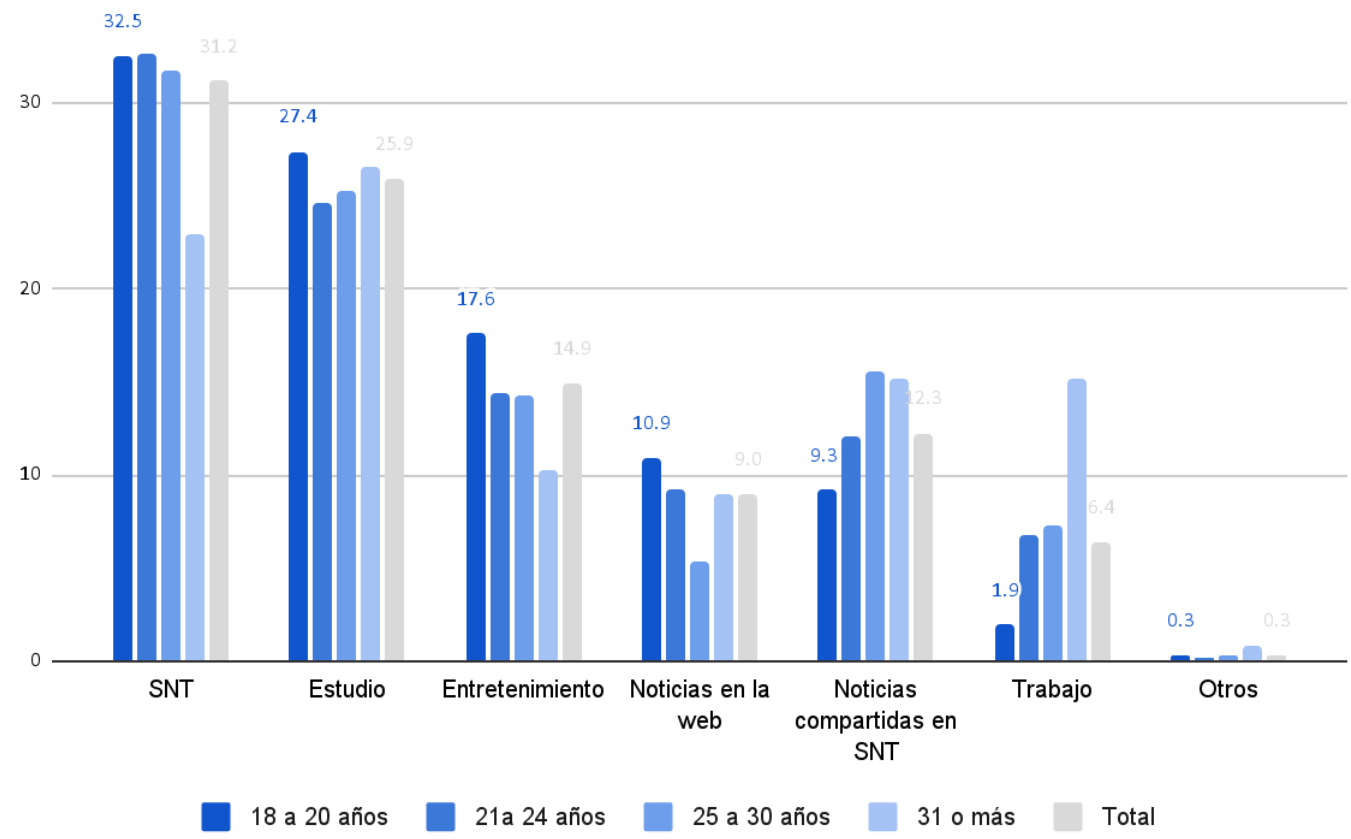

Fuente: elaboración propia.

Figura 3: Frecuencia de conexión a internet según la edad

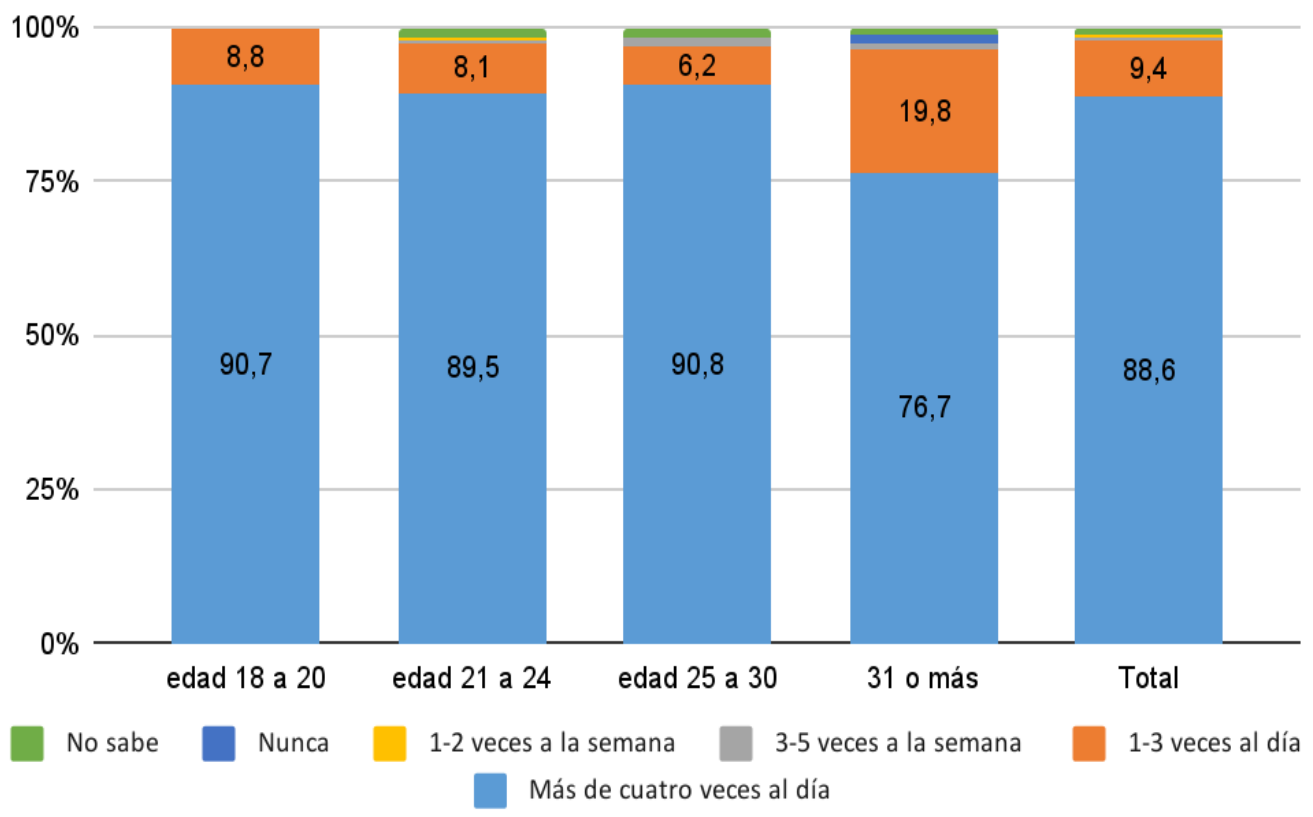

Fuente: elaboración propia. 


\subsection{Apropiación de los materiales de estudio en internet}

En cuanto al tipo de materiales que descargan los estudiantes de internet, sobre el total de menciones, un $61,7 \%$ buscó libros digitalizados; el 55,7\%, videos; el $52,2 \%$, resúmenes, y el 40,4\%, presentaciones digitales, entre otros tipos de materiales (véase figura 4). Asimismo, se observa un alto uso de videos para estudiar.

Figura 4: Materiales que buscan los estudiantes en internet para estudiar - Libros digitalizados (61.7\%)

Videos $(55.7 \%)$

Resúmenes de texto $(52.2 \%)$

- Presentaciones digitales $(40.4 \%)$

- Clases grabadas $(31.4 \%)$

Wikipedia $(29.3 \%)$

Artículos de revistas cientificas (25\%)

Apuntes de clases (20.5\%)

Trabajos de estudiantes (20.3\%)

Otros $(2.1 \%)$

No busco materiales(1.4\%)

NS/NC(1.8\%)

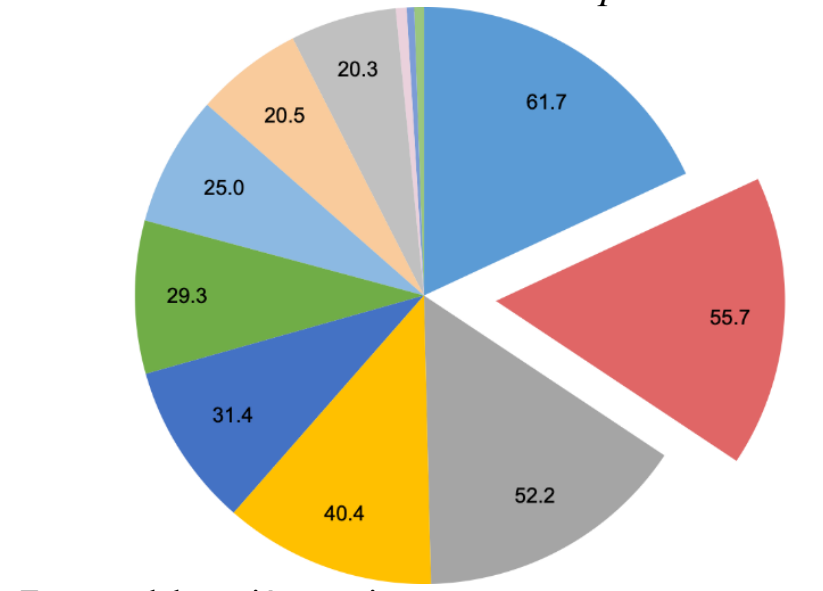

Fuente: elaboración propia.

En los grupos de discusión se confirma el acceso a tutoriales en YouTube o plataformas educativas como, por ejemplo, Educativa entre otros materiales alternativos o complementarios audiovisuales a los materiales curriculares brindados por los docentes. Según lo que los estudiantes mencionan, estos audiovisuales son útiles para comprender, fijar y clarificar temas abordados en clase. Según ellos, el material de esas plataformas permite a) contextualizar el material que se da en clase; b) profundizar en los conceptos cuando no se le entiende al docente; c) obtener una «foto de los conceptos generales», o d) seguir a docentes que publican sus clases en YouTube o en Educatina, ya que son muy claros en sus exposiciones conceptuales en relación con temas que les resultan complejos. En los grupos de discusión, como aspecto emergente, se identifica el desconocimiento que tienen los estudiantes sobre las plataformas como Timbó (portal en Uruguay que pone a disposición de sus usuarios una gran cantidad de revistas académicas en línea por medio de los principales proveedores a nivel mundial), así como de revistas especializadas en sus campos de pertinencia y 
experticia.

\subsection{Apropiaciones de SNT para acceder a materiales de estudio entre pares}

La red social WhatsApp es, sin dudas, la SNT más utilizada por los estudiantes y el principal canal por el cual establecen sus relaciones para acceder y compartir materiales de estudio (véase figura 5). Si el uso de WhatsApp es cruzado con la variable frecuencia, se observa la importante dimensión que alcanza esa plataforma dentro del mapa comunicacional de los estudiantes (véase figura 6).

Figura 5: Medios más utilizadas para compartir o comunicarse

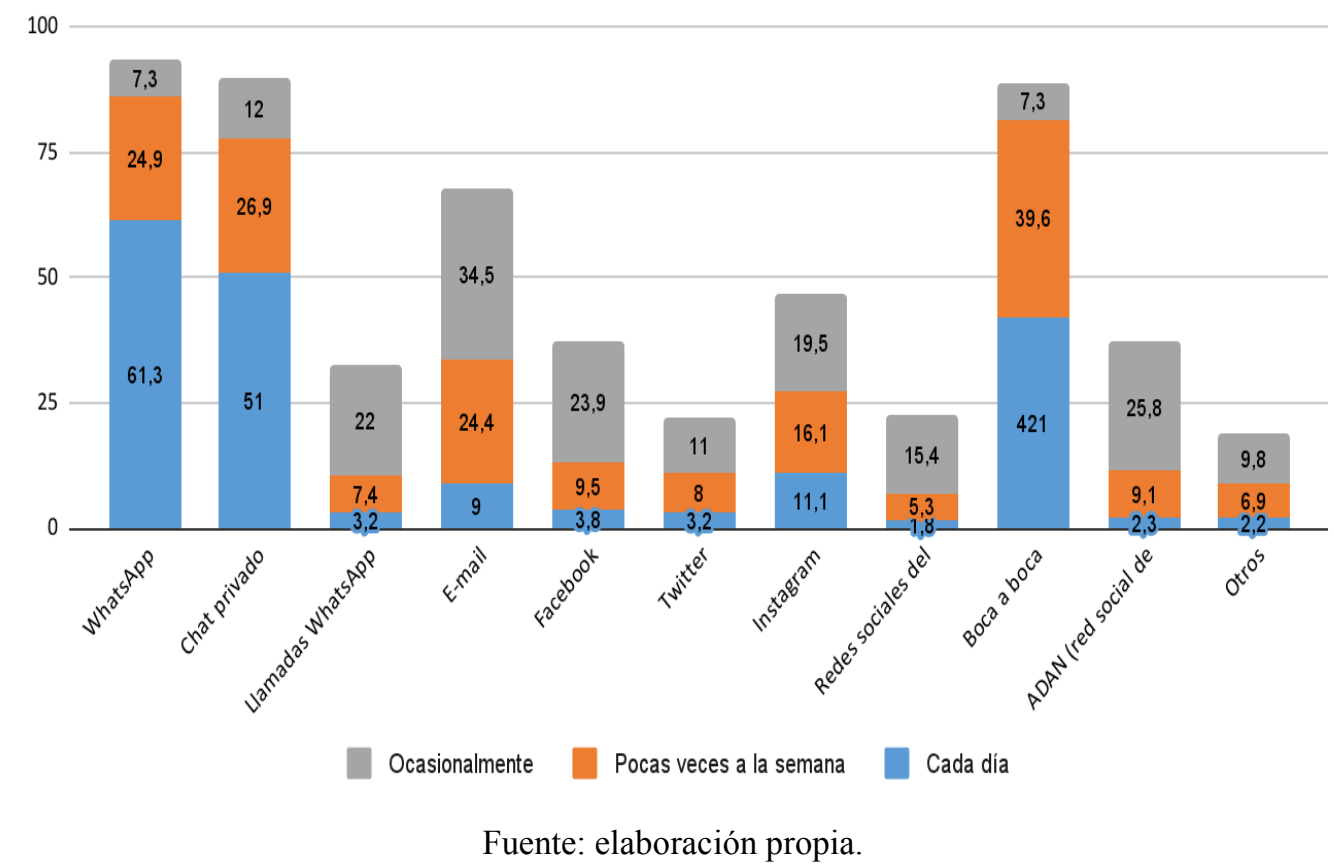


Figura 6: Frecuencia con la que comparten o se comunican entre pares según medio

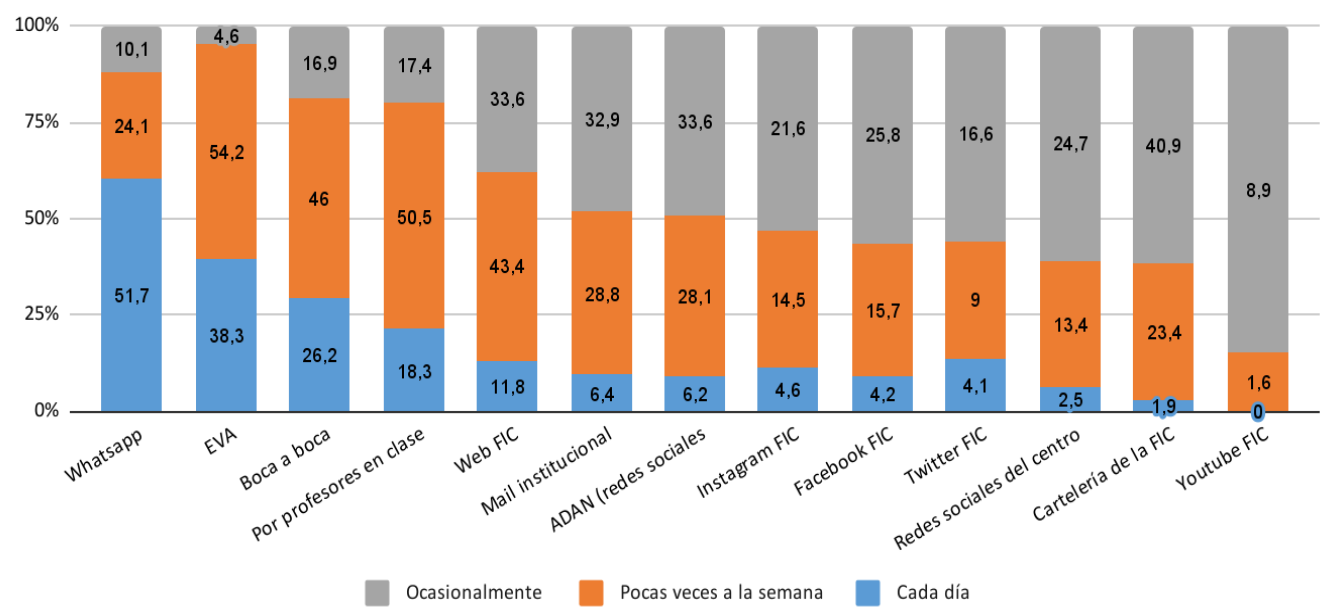

Fuente: elaboración propia.

Los datos cuantitativos y cualitativos muestran la importancia comunicacional que tiene WhatsApp dentro de la red digital de los estudiantes. En los grupos de discusión, los estudiantes mencionan que «circula todo por WhatsApp» y que por ese medio se comparte información y contenidos de carácter formal, que se obtienen de los canales y espacios de la FIC (sitio web, fotos de carteleras, tomas de pantalla de las redes sociales, audios de docentes aclarando dudas, etc.), así como también contenidos de estudio de carácter institucional (documentos de EVA, presentaciones utilizadas por los profesores para dar clase, clases grabadas de docentes, artículos de Google Académico, etc.). También mencionan que por las SNT, especialmente por WhatsApp, comparten información y contenidos informales no institucionales (enlaces de YouTube, de Educatina, resúmenes, textos no curriculares, carpetas de Google compartidas, etc.). Tales formas de apropiación de las tecnologías digitales, así como de sus sentidos y significaciones, permite a los estudiantes reducir el tiempo en la comunicación, en la búsqueda y en la producción de materiales de estudio, debido a que esa apropiación se realiza de forma colaborativa. Además, esas prácticas habilitan a los estudiantes a participar e intercambiar experiencias, saberes y demandas de forma colectiva y en busca de un mismo bien común. Las dinámicas de esas interacciones y conexiones entre pares son heterárquicas. En ellas existen múltiples accesos y múltiples vínculos, es decir, un sistema de organización social donde los individuos son entendidos como seres autónomos y en el cual sus 
relaciones son dialogantes, subrayando la revisión espontánea de su estructura, que cambia continuamente de acuerdo con las condiciones necesarias y con las innovaciones.

Los datos cuantitativos y cualitativos muestran que la plataforma EvA se utiliza principalmente como repositorio de materiales de estudio y no se observa que exista una creación colectiva o colaborativa de insumos para el estudio por parte de los estudiantes, como tampoco una significativa comunicación entre pares, como ocurre, por ejemplo, a través de WhatsApp. Los datos cuantitativos de la encuesta muestran que la mayoría de los estudiantes recurre a la plataforma institucional EVA solo con fines de consulta sobre aspectos formales curriculares: un $34,9 \%$ señala que ingresa para descargar materiales; un $29,1 \%$, para realizar tareas que suben los docentes, y un 21,8 \%, para ver calificaciones. La mediación de la comunicación que se observa en la plataforma EVA es baja: un 9,3\% de los estudiantes interactúa con los docentes, el 3,2 \% utiliza los foros de discusión y el $0,8 \%$ se pone en contacto con sus compañeros.

En la entrevista con la coordinadora de EVA se confirman los datos obtenidos en las encuestas y grupos de discusión con estudiantes, ya que señala que los docentes no han incorporado la dimensión tutorial del EVA, clave para el desarrollo de la enseñanza a través de una plataforma con fines educativos. Esto indicaría que las acciones de los docentes dentro del EVA no son pensadas con propósitos didácticos, debido a que los docentes utilizan la herramienta como repositorio y no como un dispositivo integrado en una estrategia de enseñanza. Una investigación reciente apunta que, a partir de la inclusión de las TIC a través del ingreso del EVA en la Udelar (desde el 2008), la forma de enseñar no ha cambiado significativamente y que, si bien se identifica un uso mixto (presencialvirtual), el modelo de enseñanza se centra en la presencialidad. En este marco, el estudio referido concluye que la tecnología es utilizada para habilitar contenidos más que para promover otros procesos de enseñanza (Parentelli y García, 2020).

En síntesis, las apropiaciones tecnológicas, como las prácticas y las lógicas de sentido que instauran los estudiantes en WhatsApp, parecen generar un nuevo marco de tecnosociabilidades tales como llamadas grupales, grupos de WhatsApp (por generación, asignaturas, intereses comunes, entre otros) y mensajes directos, 
así como también formas de estudio que incluyen tutoriales en línea (Educatina, YouTube, etc.), que les permiten desplegar distintas estrategias para el acceso a muchos otros saberes que complementan a los institucionales.

En relación con lo anterior, es a través de WhatsApp que los estudiantes establecen un nuevo pacto y orden tecnológico-comunicacional-informacional para instituir y constituir prácticas tecnosociales participativas, horizontales y fragmentadas, gestionadas y administradas a partir de sistemas propios de gobernanza.

\subsection{Prácticas digitales SNT de los estudiantes en la producción de materiales de estudio}

Un dato muy interesante, que surge de la encuesta y que se confirma en los grupos de discusión, es que pocos estudiantes generan productos de estudio (resúmenes, clases grabadas, slideshares, etc.) y que, quienes lo hacen, los comparten en las plataformas digitales. Del total de encuestados, un $37 \%$ no genera material, un $27 \%$ lo hace una vez cada dos o tres meses y solo un $10 \%$ lo hace cada tres o cuatro veces al mes, en promedio (véase tabla 1).

Tabla 1: Generación de material de estudio según edad

\begin{tabular}{|l|c|c|c|c|c|}
\hline $\begin{array}{l}\text { Generación de } \\
\text { materiales de estudio }\end{array}$ & $\mathbf{1 8}$ a 20 años & $\mathbf{2 1}$ a 24 años & $\mathbf{2 5}$ a 30 años & $\begin{array}{c}\mathbf{3 1} \text { años } \\
\text { o más }\end{array}$ & Total \\
\hline $3-4$ veces al mes & $7,5 \%$ & $10,9 \%$ & $13,8 \%$ & $8,1 \%$ & $10 \%$ \\
\hline $1-2$ veces al mes & $30,5 \%$ & $16,9 \%$ & $19,2 \%$ & $20,9 \%$ & $22,3 \%$ \\
\hline Cada $2-3$ meses & $27,4 \%$ & $29,4 \%$ & $25,4 \%$ & $20,9 \%$ & $27 \%$ \\
\hline No genera materiales & $32,7 \%$ & $39,1 \%$ & $36,9 \%$ & $44,2 \%$ & $37,2 \%$ \\
\hline ns/nc & $1,8 \%$ & $3,6 \%$ & $4,6 \%$ & $5,8 \%$ & $3,5 \%$ \\
\hline
\end{tabular}

Fuente: elaboración propia.

Sin embargo, de aquellos estudiantes que producen material de estudio (casi un $60 \%$ ) (véase tabla 1), sin tener en cuenta la frecuencia con que lo hacen, más de un $50 \%$ señala no compartir el material producido en las plataformas (véase tabla 2). Los datos también reflejan que los estudiantes comparten más materiales de estudio compartidos por otros estudiantes (libros, enlaces, videos, etc.) que 
materiales generados por ellos mismos. En resumen, el material producido y compartido por el $60 \%$ de los estudiantes a través de internet no es numeroso ni regular, tal como surge en los grupos de discusión. Los resultados en la generación y distribución de contenidos, al menos en la FIC, no tendrían una fuerte relevancia, tal como señala Scolari (2018) en sus últimas investigaciones, ni tampoco estarían generando prácticas de autonomía en la producción de contenidos por los usuarios comunes, como señala Castells (2010) [6]. Los datos que emergen de esta investigación muestran que la producción de contenidos no es tan importante como la producción de sentido que están generando esas plataformas, tal como lo señalan los últimos resultados de los trabajos de ArribasUrrutia, Islas-Carmona y Gutiérrez Cortés (2019), Winocur (2019), Olivera (2018), entre otros, y como se confirma en los grupos de discusión de este trabajo.

Tabla 2: ¿Utilizas las nuevas tecnologías para generar contenido académico?

\begin{tabular}{|l|c|c|c|}
\hline Frecuencia & $\begin{array}{c}\text { Preparo materiales } \\
\text { de estudio, pero no } \\
\text { los comparto (\%) }\end{array}$ & $\begin{array}{c}\text { Preparo materiales } \\
\text { de estudio y los } \\
\text { comparto (\%) }\end{array}$ & $\begin{array}{c}\text { Comparto } \\
\text { materiales que } \\
\text { encuentro (\%) }\end{array}$ \\
\hline 3-4 veces al mes & 12 & 10,1 & 16,2 \\
\hline 1-2 veces al mes & 20,9 & 22,7 & 28,2 \\
\hline Cada 2-3 al mes & 22,4 & 26,5 & 23,8 \\
\hline No genero materiales & 37,6 & 35,9 & 4,3 \\
\hline ns/nc & 7,1 & 4,7 & 100 \\
\hline Total & 100 & 100 & 27,3 \\
\hline
\end{tabular}

Fuente: elaboración propia.

Otra práctica habitual en la apropiación tecnológica por parte de los estudiantes, sobre todo en WhatsApp, es que ellos van configurando y reconfigurando sus grupos, reduciéndolos a diferentes niveles o categorías, incluyendo y excluyendo a sus integrantes en relación con intereses particulares, especificidades, afinidades, unidades curriculares compartidas, etc. Tales formas de gestión entre pares dentro de la red digital les permiten tener un flujo constante de información útil, tanto de los contenidos de estudio formales como informales, y en relación con un sistema de normas de inclusión y exclusión informacional-comunicacional [7] en pro de un bien común. 
En síntesis, los procesos de convergencia de las SNT que realizan los estudiantes de la FIC, así como la mediación de carácter formal o informal o la validación de la información, generalmente se dan a partir de formas novedosas, donde intervienen estructuras lógicas y pactos sociocomunicacionales que configuran e instituyen redes tecnocomunicacionales que habilitan otras experiencias y sentidos en la apropiación de la comunicación humana. Los datos muestran que tal apropiación tecnológica, como en el caso de WhatsApp, les permite desplegar diversas estrategias para el acceso y la difusión de contenidos de estudio, tanto formales como informales, así como facilidades en y para la circulación de los contenidos de estudio, al mismo tiempo que origina transformaciones y acomodos a las múltiples necesidades tecnosociales que indican otras formas de gestión y de socialización juvenil, más allá de las tradicionales. El complejo cambio en los procesos de tecnosociabilidad supone una incorporación muy rápida de prácticas, lógicas y relaciones en las actividades cotidianas de los estudiantes, quienes ahora transitan, habitan, conviven y comparten esos nuevos espacios tecnológicos, geográficos y sociales.

\section{Conclusiones}

Los hallazgos del estudio reportado en este artículo amplían los resultados encontrados en los trabajos mencionados anteriormente. Se observa que el cambio que introdujeron las SNT en las comunicaciones de los estudiantes de educación superior — como, por ejemplo, WhatsApp — alteró las formas de habitar y percibir el territorio, lo que supuso una incorporación muy rápida en sus vidas cotidianas de prácticas y significados sociales (tecnosociabilidad), como, por ejemplo, acceder a los materiales de estudio (formales e informales) y compartirlos entre pares.

Los estudiantes que transitan, habitan, conviven y comparten los espacios TGS, deben ahora adaptarse y entender un nuevo paradigma mediacional compuesto por novedosas formas de intervención y apropiación social, que crean, al mismo tiempo, nuevas dinámicas y lógicas de relacionamiento que no podrían entenderse sin las SNT (intercambio de roles emisores y receptores de comunicación, relaciones y participaciones en redes virtuales digitales, modos y formas de compartir, modalidades de gobernanza del territorio virtual, etc.). Las novedosas 
formas de relacionamiento y las construcciones tecnosociales emergen como consecuencia del despliegue de las nuevas tecnologías y visibilizan que internet no es solo una herramienta que permite relacionarnos de otra manera con el territorio y lo social, con el espacio, el tiempo y el lugar, sino que es una herramienta que debiera ser entendida como un espacio social en sí mismo, donde las relaciones se hacen y están presentes. Se señala en este estudio que tales formas de relación tecnosociales están habilitando nuevas extraterritorialidades, ya sean físicas o virtuales, que generan un espacio TGS que afecta a los estudiantes en la inclusión o exclusión de territorios concebidos como nuevos modos de interacción, estrategias y apropiaciones, pero no solo de recursos y materiales académicos, sino también del sentido en los procesos de aprendizaje.

La conexión a WhatsApp emerge con fuerza en este estudio, quizás como antes lo hizo Facebook, pero no como parte de la revolución tecnológica, sino, como explica Castells (2004), como un nuevo modo de relación entre los procesos simbólicos - que constituyen lo cultural- y las formas de producción y distribución de los bienes y servicios. Se entiende aquí que los nuevos espacios TGS dan cuenta no solamente de la revolución tecnológica, sino también de

a) nuevos modos de percepción y de tecnosociabilidades que habilitaron las SNT;

b) la convergencia de recursos y funcionalidades que apoyan las estrategias de enseñanza; c) otras formas de relacionamiento regladas por prácticas tecnocomunicacionales que constituyen e instituyen los estudiantes a partir de la gestión de sistemas propios de gobernanza dentro de los espacios TGS, donde se condensan nuevas lógicas de relacionamiento con el espacio, el tiempo y el lugar;

c) la articulación de saberes y aprendizajes en las SNT y del conjunto de prácticas y representaciones que median las relaciones entre la cultura y la tecnología;

d) la concepción de fenómenos de integración y exclusión informacional y comunicacional, y

e) formas de conexión heterárquicas y creativas. Lo anterior da cuenta de la 
conformación de una comunidad virtual para el aprendizaje, constituida por los espacios TGS y regulada por los mismos estudiantes, a través de la construcción colaborativa de estrategias con un fin común.

\section{Referencias bibliográficas}

Amador, P., y Amador, J. (2014). Academic advising via Facebook: Examining student help seeking. The Internet and Higher Education, 21, 9-16. doi: 10.1016/j.iheduc.2013.10.003

Arribas-Urrutia, A., Islas-Carmona, O., y Gutiérrez Cortés, F. (2019). De prosumidores a observadores: una tendencia emergente en Internet y en los jóvenes ecuatorianos. Resultados del estudio del World Internet Project, Ecuador. Revista Latina de Comunicación Social, 74, 969-996. Recuperado de http://www.revistalatinacs.org/074paper/1367/50es.html

Azizi, S. M., Soroush A., y Khatony, A. (2019). The relationship between social networking addiction and academic performance in Iranian students of medical sciences: a cross-sectional study. BMC Psychology, 7(1), 1-8. doi: $\underline{10.1186 / \mathrm{s} 40359-019-0305-0}$

Bingham, T., y Conner, M. (2015). The new social learning: Connect, collaborate, work. Alexandria: ATD Press.

Bouton, E., Bar Tal, S., y Asterhan, C. S. C. (2021). Students, social network technology and learning in higher education: Visions of collaborative knowledge construction vs. the reality of knowledge sharing. The Internet and Higher Education, 49. doi: 10.1016/j.iheduc.2020.100787

Brand, M., Young, K. S., Laier, C., Wölfling, K., y Potenza, M. N. (2016). Integrating psychological and neurobiological considerations regarding the development and maintenance of specific internet-use disorders: An interaction of person-affect-cognition-execution (I-PACE) model. Neuroscience y Biobehavioral Reviews, 71, 252-266, doi: 10.1016/j.neubiorev.2016.08.033

Cardozo, J. (setiembre, 2010). TIC y educación: los aprendizajes colaborativos como estrategia para los procesos de construcción de conocimiento. Trabajo presentado en el Congreso Iberoamericano en Educación, Buenos Aires, Argentina. Recuperado de https://www.adeepra.org.ar/congresos/Congreso\%20IBEROAMERICAN O/TICEDUCACION/RLE2284_Cardozo.pdf 
Castells, M. (2004). The Information Age: Economy, Society and Culture (Vol. II, 2. ${ }^{a}$ ed.). Oxford, Inglaterra: Blackwel Publishing Lts.

Castells, M. (2010). Comunicación y poder. Madrid: Alianza Editorial.

Cobo, C. (2016). La innovación pendiente: reflexiones (y provocaciones) sobre educación, tecnología y conocimiento. Montevideo: Sudamericana . Recuperado de https://digital.fundacionceibal.edu.uy/jspui/bitstream/123456789/159/1/La innovacion pendiente.pdf

Collazo, C. A., y Mendoza, J. (2006). Cómo aprovechar el «aprendizaje colaborativo» en el aula. Educación y Educadores, 9(2), 61-76, Recuperado de https://www.redalyc.org/pdf/834/83490204.pdf

Collins, A., y Halverson, R. (2009). Rethinking education in the age of technology: the digital revolution and the schools. Nueva York: Teachers College.

Dabbagh, N., y Kitsantas, A. (2012). Personal learning environments, social media, and self-regulated learning: A natural formula for connecting formal and informal learning. The Internet and Higher Education, 15(1), 3-8. doi: 10.1016/j.iheduc.2011.06.002

DeAndrea, D. C., Ellison, N. B., LaRose, R., Steinfield, C., y Fiore, A. (2012). Serious social media: On the use of social media for improving students' adjustment to college. The Internet and Higher Education, 15(1), 15-23. doi: $\underline{10.1016 / \text { j.iheduc.2011.05.009 }}$

Dillenbourg P. (1999) What do you mean by collaborative learning? En P. Dillenbourg (Ed.), Collaborative-learning: Cognitive and Computational Approaches (pp. 1-19). Oxford: Elsevier. Recuperado de https://telearn.archives-ouvertes.fr/hal-00190240/document

Dillenbourg, P., y Baker, M. J. (junio, 1996). Negotiation Spaces in HumanComputer Collaboration. Trabajo presentado en Coop'96, Second International Conference on Design of Cooperative Systems, Inria, Juanles-Pins, Francia.

García, J. (2000). La evolución de las organizaciones: de la jerarquía a la heterarquía. Universidad de Oviedo.

Gairín, J. (2006). Las comunidades virtuales de aprendizaje. Educar, 37, 4164. Recuperado de http://www.redalyc.org/articulo.oa? $\mathrm{id}=342130826004$ 
Greenhow, C., y Robelia, B. (2009a). Informal learning and identity formation in online social networks. Learning, Media and Technolology, 34(2), 119140. doi: $\underline{10.1080 / 17439880902923580}$

Greenhow, C., y Robelia, B. (2009b). Old communication, new literacies: Social network sites as social learning resources. Journal of Computer-Mediated Communication, 14(4), 1130-1161. doi: 10.1111/j.1083-

$\underline{6101.2009 .01484 . \mathrm{x}}$

Hedlund, G., y Nonaka, I. (1993). Models of knowledge management in the West and Japan. En P. Lorange, B. Chakravarthy, J. Roos y H. Van de Ven (Eds.), Implementing Strategic Processes, Change, Learning and Cooperation (pp. 117-144). Londres: Macmillan.

Hew, K. F. (2011). Students' and teachers' use of Facebook. Computers in Human Behavior, 27(2), 662-676. doi: 10.1016/j.chb.2010.11.020

Junco, R. (2012). Too much face and not enough books: The relationship between multiple indices of Facebook use and academic performance. Computers in Human Behavior, 28(1), 187-198. doi: 10.1016/j.chb.2011.08.026

Junco, R., y Cotten, S. R. (2012). No A 4 U: The relationship between multitasking and academic performance. Computers and Education, 59(2), 505-514. doi: 10.1016/j.compedu.2011.12.023

Kadushin, C. (2004). Some Basic Network Concepts and Propositions. En Introduction to Social Network Theory. Recuperado de http://www.cin.ufpe.br/ rbcp/taia/Kadushin_Concepts.pdf

Kolhar, M., Raisa, A., y Abdalla, A. (2021). Effect of social media use on learning, social interactions, and sleep duration among university students. Saudi Journal of Biological Sciences, 28(4), 2216-2222. doi: $\underline{10.1016 / j . s j b s .2021 .01 .010}$

Kontopoulos, K. (1993). The Logics of Social Structure. Cambridge: Cambridge University Press. doi: 10.1017/CBO9780511570971

Laru, J., Näykki, P., y Järvelä, S. (2012). Supporting small-group learning using multiple Web 2.0 tools: A case study in the higher education context. The Internet and Higher Education, 15(1), 29-38. doi:

$\underline{10.1016 / j . i h e d u c .2011 .08 .004}$

Livingstone, S., y Sefton-Green, J. (2016). The Class: Living and Learning in the Digital Age. New York University Press. 
McCulloch, W. (1945). Una heterarquía de valores determinada por la topología de las redes nerviosas. Boletín de Biofísica Matemática, 7, 89-93.

Martín-Barbero, J. (2006). La razón técnica desafía a la razón escolar. En M. Narodowski, H. Ospina y A. Martínez Boom (Comps.), La razón técnica desafía a la razón escolar. Buenos Aires: Noveduc.

Molinillo, S., Anaya-Sánchez, R., Aguilar-Illescas, R., y Vallespín-Arán, M. (2018). Social media-based collaborative learning: Exploring antecedents of attitude. The Internet and Higher Education, 38, 18-27. doi: $\underline{10.1016 / j . i h e d u c .2018 .04 .003}$

Montag, C., Zhao, Z., Sindermann, C., Xu, L., Fu, M., Zheng, X., Li, K., Kendrick, K. M., Dai, J., y Becker, B. (2018). Internet Communication Disorder and the structure of the human brain: initial insights on WeChat addiction. Scientific Reports, 8(2155). doi: 10.1038/s41598-018-19904-y

Moore, M. G. (1989). Editorial: Three types of interaction. The American Journal of Distance Education, 3(2), 1-7. doi: 10.1080/08923648909526659

Naciones Unidas, Cepal (2015). Estado de la banda ancha en América Latina y el Caribe. Recuperado de https://www.cepal.org/es/publicaciones/38605estado-la-banda-ancha-america-latina-caribe-2015

Olivera, M. N. (2013). E-Migration: a new configuration of technological, geographical and social spaces. International Journal of E-Politics (IJEP), 4(1), 18-31. doi: 10.4018/jep.2013010102

Olivera, M. N., Morales, M. J., Passarini, A., y Correa, N. (2017). Plataformas virtuales: ¿herramientas para el aprendizaje? Las diferencias entre ADÁN y EVA. Comunicação, Mídia e Consumo. 14(40), 90-109. doi: $\underline{10.18568 / C M C . V 14 I 40.1308}$

Olivera, M. N. (julio-agosto, 2018). Playlist, un experimento comunicacional multiplataforma: ¿nuevos hábitos de consumo y participación en cuarta pantalla? Trabajo presentado en el XIV Congreso Latinoamericano de Investigadores de la Comunicación, San José, Costa Rica. Recuperado de http://alaic2018.ucr.ac.cr/sites/default/files/2019-02/GT\%2010\%20\%20ALAIC\%202018.pdf

Olivera, M. N. (2019). ¿La política de Educación Superior es una oportunidad de Integración Regional, Tecnológica, Geográfica y Social (TGS)?: el caso de la Licenciatura Binacional de Turismo (Argentina y Uruguay). Dilemas Contemporáneos: Educación, Política y Valores, 6(3), México. 
Olivera, M. N. (Coord.) (2020a). Universidad y TIC: análisis de las nuevas prácticas de producción y circulación de la información del Cenur Litoral Norte. Montevideo: CSIC, Udelar. Recuperado de https://www.litoralnorte.udelar.edu.uy/images/documentos/I MAS D ebo ok.pdf

Olivera, M. N. (Coord.) (2020b). Universidad y TIC: ¿nuevos espacios tecnológicos y sociales de aprendizaje? Recuperado de https://www.sic.uy/pimceu/

Olivera, N. M. (2021). Universidad y WhatsApp: cuando los jóvenes reconfiguran y tensionan los espacios comunicacionales. Manuscrito en proceso.

Pataraia, N., Falconer, I., Margaryan, A., Littlejohn, A., y Fincher, S. (2014). $¿$ Con quién hablas de tu docencia?: actividades de networking entre profesores universitarios. Investigación de Aprendizaje de Primera Línea, 2(2), 4-14.

Pardo, H., y Cobo, C. (2020). Expandir la universidad más allá de la enseñanza remota de emergencia: ideas hacia un modelo híbrido post-pandemia. Barcelona: Outliers School. Recuperado de https://outliersschool.net/wpcontent/uploads/2020/05/Expandir_la_universidad.pdf

Parentelli, V., y García, S. (2020). Uso de entornos virtuales en los procesos de enseñanza y aprendizaje en la formación de grado. Manuscrito inédito, Facultad de Información y Comunicación, Universidad de la República, Montevideo, Uruguay.

Piotrowski, C. (2015). Scholarly research on educational adaptation of social media: Is there evidence of publication bias? College Student Journal, 49, $447-451$.

Radar. (2019). El internauta uruguayo. Encuesta 2019. Montevideo: Grupo Radar. Recuperado de https://gruporadarllc.com/shop/informes/26informe- 0. html

Radar. (2020). El internauta uruguayo. Encuesta 2020. Montevideo: Grupo Radar. Recuperado de https://www.gruporadar.com.uy/blog/perfil-delinternauta-uruguayo-2020

Rodés, V., Gewerc-Barujel, A., y Llamas-Nistal, M. (2019). University Teachers and Open Educational Resources: Case Studies from Latin America. The International Review of Research in Open and Distributed Learning, 20(1). doi: 10.19173/irrodl.v20i1.3853 
Scolari, C. A. (2018). Adolescentes, medios de comunicación y culturas colaborativas: aprovechando las competencias transmedia de los jóvenes en el aula. Barcelona: H2020-Research and Innovation Actions. Recuperado de $\underline{\text { https://digital.fundacionceibal.edu.uy/jspui/handle/123456789/247 }}$

Selwyn, N., y Stirling, E. (2016). Social media and education... now the dust has settled. Learning, Media and Technology, 41(1), 1-5.

Sha, P., Sariyska, R., Riedl, R., Lachmann, B., y Montag, C. (2019). Linking Internet Communication and Smartphone Use Disorder by taking a closer look at the Facebook and WhatsApp applications. Addictive Behaviors Reports, 9. doi: 10.1016/j.abrep.2018.100148

Silvio, J. (noviembre, 1999). Las comunidades virtuales como conductoras del aprendizaje permanente. Sistemas de Aprendizaje Virtual. Simposio organizado por la Red Iberoamericana de Informática Educativa (Ribie), el Centro Internacional de Educación y Desarrollo (CIED) de la Compañía Petróleos de Venezuela y el Iesalc/Unesco, Caracas. Recuperado de http://cmapspublic3.ihmc.us/rid=1H30ZDCYS-1HRGM7BQV6/Comunidades $\% 20$ Virtuales $\% 20$ como $\% 20$ Conductoras $\% 20$ del $\% 20 \mathrm{~A}$ prendizaje $\% 20$ Permanente.pdf

Smith, E. (2016). A real double-edged sword: Undergraduates perceptions of social media in their learning. Computers \& Education, 103, 44-58. doi: $\underline{10.1016 / \text { j.compedu.2016.09.009 }}$

Universidad de la República, Dirección General de Planeamiento. (2019a). Estadísticas básicas 2019. Recuperado de https://planeamiento.udelar.edu.uy/wpcontent/uploads/sites/33/2020/12/web_Estadisticas-basicas 2019.pdf

Universidad de la República, Dirección General de Planeamiento. (2019b). Perfil de los estudiantes de grado. Recuperado de http://gestion.udelar.edu.uy/planeamiento

Universidad de la República, Comisión Sectorial de Enseñanza, programa de Entornos Virtuales de Aprendizaje. (2020). Análisis de la utilización del Entorno Virtual de Aprendizaje de la Udelar durante el COVID-19. Recuperado de https://proeva.udelar.edu.uy/analisis-de-la-utilizacion-del$\underline{\text { entorno-virtual-de-aprendizaje-de-la-udelar-durante-el-covid-19/ }}$

Wegerif, R. (2012). Dialogic: Education for the internet age. Nueva York: Routledge. 
Winocur, R. (2019). La tribu de los memes: un territorio virtual de inclusiónexclusión entre los adolescentes. Comunicación y Sociedad, año 16, 1-22. Recuperado de http://www.comunicacionysociedad.cucsh.udg.mx/index.php/comsoc/artic $\underline{\text { le/view/7327 }}$

Wodzicki, K., Schwämmlein, E., y Moskaliuk, J. (2012). «Actually, I wanted to learn»: Study-related knowledge exchange on social networking sites. The Internet and Higher Education, 15(1), 9-14. doi:

$\underline{10.1016 / \mathrm{j} . \text { iheduc.2011.05.008 }}$

Yu, A. Y., Tian, S. W., Vogel, D., y Kwok, R. C. W. (2010). Can learning be virtually boosted?: An investigation of online social networking impacts. Computers y Education, 55(4), 1494-1503. doi: 10.1016/j.compedu.2010.06.015

Zañartu, L. (2003). Aprendizaje colaborativo: una nueva forma de diálogo interpersonal y en red. Revista Digital de Educación y Nuevas Eecnologías, 5(28). Recuperado de http://files.enriquecereducaciontic.webnode.es/200000026-9a1009c069/Contexto_Educativo_Revista_digital_de_Educacion_y_Nuevas_Tecnologias.pdf

\section{Notas}

[1] Según la última Encuesta de Usos de Tecnología de Información y Comunicación (Eutic) 2019, realizada por la Agencia de Gobierno Electrónico y Sociedad de la Información y Comunicación (Agesic) del Gobierno uruguayo, nueve de cada diez hogares en el Uruguay acceden a internet, de los cuales el $71 \%$ tiene banda ancha. Esta encuesta indica, además, que la brecha socioeconómica en las tasas de conectividad ha disminuido sustancialmente, reduciéndose de 66 puntos porcentuales en el año 2010 a 11 en 2019. Asimismo, en el informe 2019 sobre el mercado de las telecomunicaciones en el Uruguay, realizado por la Unidad Reguladora de Servicios de Comunicaciones (Ursec), se señala que hay 941806 conexiones fijas residenciales, sobre un total de 1160000 hogares, y que, de ellas, el 69 \% son por fibra óptica. El informe Estado de la banda ancha en América Latina y el Caribe 2015 de la Comisión Económica para América Latina (Cepal) señala que Uruguay es el país con mayor velocidad de conexión, similar a países europeos como España, Portugal e Inglaterra (Naciones 
Unidas, Cepal, 2015).

[2] Para mayores especificaciones sobre el concepto de SNT, consultar Kadushin, (2004) y Pataraia, Falconer, Margaryan, Littlejohn y Fincher (2014).

[3] El concepto de heterarquía comenzó a ser usado por algunos autores (Livingstone y Sefton-Green, 2016; Kontopoulos, 1993; Hedlund y Nonaka, 1993; García, 2000) y se aplicó a un nuevo orden en las redes, un sistema de organización social donde los individuos son entendidos como seres autónomos, autoinventados, y en el cual sus relaciones son dialogantes, subrayando la revisión continua y espontánea de su estructura, que cambia continuamente de acuerdo con las condiciones necesarias y las innovaciones.

[4] Refiere a la implementación de la encuesta en reiteradas veces en distintas asignaturas de modo de poder alcanzar el mayor número de respuestas.

[5] Estrategia cualitativa no probabilística para alcanzar mayor participación.

[6] Castells entiende que la difusión de internet y de la comunicación inalámbrica apoya y refuerza las prácticas de autonomía, como cargar en la red contenidos producidos por los usuarios. Sin embargo, los resultados de este trabajo de investigación en Uruguay y dentro del contexto universitario no evidencian dicha afirmación basada en estudios de la población catalana (2010, p. 181).

[7] Por ejemplo, una de las dimensiones de la exclusión o inclusión de estudiantes en los grupos de WhatsApp está relacionada con el tipo de prácticas de estudio que realizan los mismos estudiantes, tales como pedir o estudiar de resúmenes, seleccionar con qué grupos compartir material propio de estudio, ajustar el territorio virtual para habitarlo solo con aquellos compañeros que «son serios» para estudiar, etc. La integración de los estudiantes en los grupos de WhatsApp se maneja de acuerdo al criterio de territorios comunes: generacional, por asignatura, contenidos, afinidades, etcétera.

\section{Nota de los autores}

Financiamiento: Este trabajo fue financiado por la Comisión Sectorial de Enseñanza y la Comisión Sectorial de Investigación Científica de la Universidad de la República en el marco de la convocatoria a proyectos de investigación para 
la mejora de la calidad de la enseñanza universitaria (PIMCEU) del año 2017.

\section{Notas del editor}

El presente manuscrito fue aprobado para su publicación por Mario Barité.

La corrección de estilo del presente texto fue realizada por Katherine Chamyan en el marco del convenio celebrado entre la FHCE (Tecnicatura Universitaria en Corrección de Estilo-Facultad de Humanidades y Ciencias de la Educación) y la FIC (Facultad de Información y Comunicación, Universidad de la República).

\section{Nota de contribución autoral}

E1 60\% fue desarrollado por Mauricio Olivera y el 40\% Varenka Parentelli. 